\title{
SOME IMMERSION THEOREMS FOR MANIFOLDS
}

\author{
BY \\ A. DUANE RANDALL
}

\begin{abstract}
In this paper we obtain several results on immersing manifolds into Euclidean spaces. For example, a spin manifold $M^{n}$ immerses in $R^{2 n-3}$ for dimension $n \equiv 0 \bmod 4$ and $n$ not a power of 2 . A spin manifold $M^{n}$ immerses in $R^{2 n-4}$ for $n \equiv 7 \bmod 8$ and $n>7$. Let $M^{n}$ be a 2-connected manifold for $n \equiv 6 \bmod 8$ and $n>6$ such that $H_{3}(M ; Z)$ has no 2-torsion. Then $M$ immerses in $R^{2 n-5}$ and embeds in $R^{2 n-4}$. The method of proof consists of expressing $k$-invariants in Postnikov resolutions for the stable normal bundle of a manifold by means of higher order cohomology operations. Properties of the normal bundle are used to evaluate the operations.
\end{abstract}

1. Preliminaries. By a manifold $M^{n}$ we mean that $M$ is a closed connected smooth manifold of dimension $n$. We write $M \subseteq R^{s}$ and $M \subset R^{t}$ to denote the existence of a differentiable immersion of $M$ into Euclidean $s$-space and a smooth embedding of $M$ into Euclidean $t$-space respectively. A manifold $M$ is called a spin manifold iff $w_{1}(M)=w_{2}(M)=0$. The geometric dimension of a stable vector bundle $\xi$ over a complex $X$, denoted $\operatorname{g} \cdot \operatorname{dim} \xi$, is the smallest integer $k$ for which there is a $k$-plane bundle over $X$ stably isomorphic to $\xi$. The coefficient group for singular cohomology is understood to be $Z_{2}$ whenever omitted. We denote the mod 2 Steenrod algebra by $A . A(Y)$ denotes the semitensor algebra $H^{*}(Y) \otimes A$ defined in [19] for any space $Y$. Finally $\alpha(n)$ represents the number of 1's appearing in the dyadic expansion of the positive integer $n$. In [10] Glover proves that a $k$-connected manifold $M^{n}$ embeds in $R^{2 n-2 k}$ if it immerses in $R^{2 n-2 k-1}$ for $0 \leqq k \leqq(n-3) / 4$. All spaces are assumed to be complexes (pathwise connected $\mathrm{CW}$-complexes with basepoint) and all maps preserve basepoint. The author wishes to express his sincere gratitude to his advisor, Professor Emery Thomas.

A formulation of [18, Theorem II] for spin manifolds is the following

Proposition 1.1. Let $M^{n}$ be a spin manifold such that $\bar{w}_{n-k}(M) \neq 0$. There are nonnegative integers $a_{j}$ for $j \geqq 0$ satisfying the conditions:

1. $\sum a_{j}=k$,

2. $\sum 2^{j} a_{j}=n$,

3. $a_{1}$ is even,

4. if $a_{0}=0$, the first nonzero $a_{j}$ and its immediate successor $a_{j+1}$ must be even,

5. if $a_{2}$ is even, $a_{1} \equiv 0 \bmod 4$; if $a_{2}$ is odd, $a_{1} \equiv 2 \bmod 4$.

Received by the editors May 8, 1969.

AMS 1970 subject classifications. Primary 57D40, 55G45, 55G20, 55G40.

Key words and phrases. Immersion, embedding, spin manifold, normal bundle, Postnikov resolution, $k$-invariant, twisted cohomology operation, higher order cohomology operation, Thom complex, Poincaré duality, Dold manifold.

Copyright (C) 1971, American Mathematical Society 
Proof. Massey and Peterson show in [18] that there exists an admissible monomial $S q^{I}$ in $A$ of degree $n-k$ such that $S q^{I} x \neq 0$ for some class $x$ in $H^{k}(M)$. They write $S q^{I}=S q^{i_{1}} \cdots S q^{i_{p}}$ and set $a_{j}=i_{j}-2 i_{j+1}$ for $0<j<p$ and $a_{p}=i_{p}$. Since $M$ is a spin manifold, the Wu classes $V_{s}(M)=0$ for $0<s<4$. So by the Adem relations $S q^{t}: H^{n-t}(M) \rightarrow H^{n}(M)$ is trivial for $t$ not divisible by 4 . Thus $i_{1} \equiv 0 \bmod 4$ and condition 5 follows. Conditions 1-4 are established in [18].

COROLlaRY 1.2. Let $M^{n}$ be a spin manifold with $n \equiv j \bmod 8$ for $n>j$ and $3<j<8$. Then $\bar{w}_{n-j}(M)=0$.

THEOREM 1.3. Let $M^{n}$ be a 3-connected manifold with $n \equiv j \bmod 8, n>j$, and $3<j<8$. Then $M \subset R^{2 n-4}$ and $M \subseteq R^{2 n-5}$.

Proof. Now $\bar{w}_{n-j}(M)=0$ by (1.2) so $M \subset R^{2 n-4}$ from [11, Theorem 2.3]. Let $v$ denote the normal bundle to an embedding of $M$ in $R^{2 n-4}$. Note that $\pi_{n-1}\left(S^{n-5}\right)$ is the 4-stem and so is trivial. Thus the only obstruction to a cross-section of the sphere bundle associated to $v$ is the Euler class $\chi(v)$. But $\chi(v)=0$ since $v$ is the normal bundle to an embedding. So $M \subseteq R^{2 n-5}$ by Hirsch [12].

REMARK. Set $m=2^{r}+1$ for $r>1$. Quaternionic projective space $Q P^{m} \notin R^{8 m-5}$ from [22] and $Q P^{m} \ddagger R^{8 m-6}$ from [7] so (1.3) is a best possible result.

Proposition 1.4. Let $K$ be a complex with dimension $n \equiv 6 \bmod 8$ and $n>14$. Suppose that $H^{n-i}(K)=0$ for $1 \leqq i \leqq 4$. Let $\xi$ be any stable orientable bundle over $K$. Then g.dim $\xi \leqq n-7$ iff $w_{n-6}(\xi)=0$.

Proof. Write $n=8 t+6$ for $t>1$ and let the map $\xi: K \rightarrow B S O$ classify the bundle $\xi$. The homotopy groups of the fiber $V$ for the fibration $\pi: B S O(8 t-1) \rightarrow B S O$ are listed in [13]. In particular, $\pi_{8 t-1}(V)=Z_{2}$ while $\pi_{8 t}(V)=\pi_{8 t+5}(V)=0$. Thus $\xi$ lifts to $B S O(8 t-1)$ iff $w_{8 t}(\xi)=0$.

THEOREM 1.5. Let $M^{n}$ be a 4-connected manifold with $n \equiv 6 \bmod 8$ and $n>14$. Then $M \subseteq R^{2 n-7}$ and $M \subset R^{2 n-6}$.

Proof. Let $v$ denote the stable normal bundle of $M$. By (1.2) $w_{n-6}(v)=0$. Poincaré duality gives $H^{n-i}(M)=0$ for $1 \leqq i \leqq 4$. Thus $M \subseteq R^{2 n-7}$ from (1.4) and [12]. Finally $M \subset R^{2 n-6}$ by Glover [10].

2. Cohomology operations and $k$-invariants. In [25] Thomas describes a method for expressing $k$-invariants in a Postnikov resolution for a fibration in terms of higher order cohomology operations applied to classes coming from the base of the fibration. We consider only a Postnikov resolution for the fibration $\pi: B_{m} \rightarrow B$ through dimensions $\leqq t$ where $\pi^{*}$ is surjective and $m<t<2 m$. Fiere $B_{m}$ and $B$ denote either $B S O(m)$ and $B S O$ or $B$ Spin $(m)$ and $B$ Spin respertively. We derive from the generating class theorem [25, Theorem 5.9] a version for the case of independent second-order $k$-invariants in a resolution for $\pi$. Consider the following commutative diagram. 


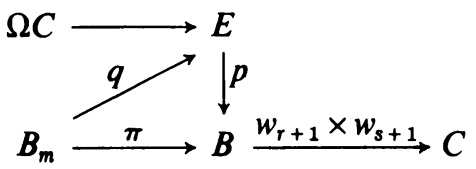

Here $E$ represents the first stage in a resolution for $\pi$ through dimensions $\leqq t$ and so $p$ is the principal fiber map classified by the vector $\left(w_{r+1}, w_{s+1}\right)$ of StiefelWhitney classes. Let $\iota_{r}$ denote the fundamental class of the factor $K\left(Z_{2}, r\right)$ in $\Omega C$ and define $\iota_{s}$ similarly. Let $m: \Omega C \times E \rightarrow E$ denote multiplication in the principal fibration and $\rho: \Omega C \times E \rightarrow E$ the projection map. Suppose that a class $k$ in $H^{p}(E)$ for $m<p \leqq t$ is a second-order $k$-invariant for $\pi$ independent of $w_{s+1}$. That is, $\mu(k)=\hat{\alpha} \circ\left(\iota_{r} \otimes 1\right)$ for some class $\hat{\alpha}$ in $A(B)$. The morphism $\mu: H^{*}(E) \rightarrow H^{*}(\Omega C \times E, E)$ is defined in [25] so that $j^{*} \circ \mu=m^{*}-\rho^{*}$ for the inclusion $j: \Omega C \times E \rightarrow(\Omega C \times E, E)$. From construction of the resolution for $\pi, \operatorname{ker} p^{*}=\operatorname{ker} \pi^{*}$ through dimensions $\leqq t$ so $\operatorname{ker} q^{*} \cap \operatorname{ker} \mu=0$ through dimensions $\leqq t$ by [25, Proposition 5.11].

We suppose also that a class $v$ in $H^{*}(B)$ is a generating class for $k$. That is, there is a complex $K$, a map $\eta: B \rightarrow K$, and a class $\alpha$ in $A(K)$ such that $\hat{\alpha}=\eta^{*} \alpha$. There are vectors $\beta=\left(\beta_{1}, \ldots, \beta_{j}\right)$ and $\psi=\left(\psi_{1}, \ldots, \psi_{j}\right)$ of primary operations over $A(K)$ and a primary operation $\varphi$ over $A(K)$ such that $(\varphi, \psi) v=\left(w_{r+1}, 0\right)$ and there is a relation

$$
\alpha \cdot \varphi+\beta \cdot \psi=0 .
$$

Let $\Omega$ be any secondary operation associated to (2.2). Then $\Omega$ determines a coset $L$ of $\operatorname{Indet}^{p}(B ; \Omega, \eta)$ such that $\Omega\left(\pi^{*} v, \pi^{*} \eta\right)=\pi^{*} L$. Under the above assumptions the generating class theorem states

THEOREM 2.3. $k \in \Omega\left(p^{*} v, p^{*} \eta\right)-p^{*} L$.

Proof. Consider the following commutative diagram of complexes and maps.

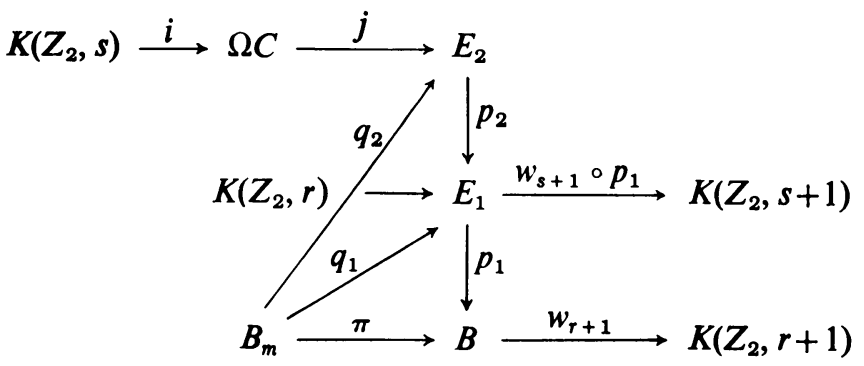

Let $\mu_{1}: H^{*}\left(E_{1}\right) \rightarrow H^{*}\left(K\left(Z_{2}, r\right) \times E_{1}, E_{1}\right)$ and $\mu_{2}: H^{*}\left(E_{2}\right) \rightarrow H^{*}\left(K\left(Z_{2}, s\right) \times E_{2}, E_{2}\right)$ be the morphisms corresponding to $\mu$ for the principal fiber maps $p_{1}$ and $p_{2}$ respectively. Identify $E_{2}$ with $E$ in (2.1) as fiber spaces over $B$ via a fiber-preserving homeomorphism and regard the composite map $p_{1} \circ p_{2}$ as $p$. Let $k_{1}$ in $H^{p}\left(E_{1}\right)$ be a class such that $\mu_{1}\left(k_{1}\right)=\hat{\alpha}(\iota \otimes 1)$ where $\iota$ is the fundamental class of $K\left(Z_{2}, r\right)$. Note that $\mu\left(p_{2}^{*} k_{1}\right)=\hat{\alpha}\left(\iota_{r} \otimes 1\right)$ so $p_{2}^{*} k_{1}=k$. (See [25] or [30].) There is a class $h$ in $\operatorname{ker} q_{1}^{*} \cap \operatorname{ker} \mu_{1} \cap H^{p}\left(E_{1}\right)$ such that $k_{1}+h \in \Omega\left(p_{1}^{*} v, p_{1}^{*} \eta\right)-p_{1}^{*} L$ by [25, Theorem 
5.9]. Since $p_{2}^{*} h \in \operatorname{ker} q^{*} \cap \operatorname{ker} \mu \cap H^{p}(E)=0$, the result follows by naturality of $\Omega$.

REMARK 1 . Let $\xi: X \rightarrow B$ classify a stable vector bundle $\xi$ over a complex $X$ for which $w_{r+1}(\xi)=w_{s+1}(\xi)=0$. Then $\xi$ lifts to $E$ in $(2.1)$ and, by definition, $k(\xi)$ $=\bigcup_{g} g^{*} k$ where $g$ ranges over all liftings of $\xi$. Note that $k(\xi)$ is a coset of the subgroup $\left(\hat{\alpha} H^{*}(X)\right) \cap H^{p}(X)$, the indeterminacy subgroup of $k(\xi)$. If $0 \in \Omega\left(\pi^{*} v, \pi^{*} \eta\right)$ and $\operatorname{Indet}^{p}\left(X ; \Omega, \xi^{*} \eta\right)=$ indeterminacy subgroup of $k(\xi)$, then $k(\xi)=\Omega\left(\xi^{*} v, \xi^{*} \eta\right)$ from (2.3).

REMARK 2. In applications of (2.3) in $\S 3$ and $\S 4$ the class $v$ is the Stiefel-Whitney class $w_{p}$ for $p$ an even integer. Suppose an operation $\Omega$ associated to relation (2.2) can be chosen 1-trivial if $B=B S O$ or spin trivial if $B=B$ Spin. (See [26].) Let $i: B_{p} \subset B_{m}$ denote the standard inclusion. It follows that $\Omega\left(\pi^{*} v, \pi^{*} \eta\right) \cap \operatorname{ker} i^{*} \neq \varnothing$ in $H^{p}\left(B_{m}\right)$ for this choice of $\Omega$ from [26, Theorem 3.3].

Versions of Thomas' generating class theorem for expressing a $k$-invariant lifted to the Thom complex of a bundle by means of an Adams-Maunder operation applied to the Thom class are given in [27], [28], and [21]. An application in $\S 4$ uses a stable tertiary operation which we define here. Consider the following stable integral relations and associated secondary operations for $t>1$.

$$
\Omega_{1}: S q^{2} S q^{4 t+2}=0, \quad \Omega_{2}:\left(S q^{2} S q^{1}\right) S q^{4 t+2}+S q^{1} S q^{4 t+4}=0 .
$$

Let $\Omega$ denote the 2-valued secondary operation $\left(\Omega_{1}, \Omega_{2}\right)$. By [16] $\Omega_{1}$ and $\Omega_{2}$ can be chosen so that $\iota \cdot S q^{2} \iota \in \Omega_{1}(\iota)$ and $0 \in \Omega_{2}(\iota)$ where $\iota$ denotes the fundamental class of $K(Z, 4 t+1)$. For this choice of $\Omega[17$, Lemma 3.1] states that the relation $S q^{2} \Omega_{1}+S q^{1} \Omega_{2}=S q^{4 t+3} S q^{2}$ holds stably and with zero indeterminacy between the component operations $\Omega_{i}$ of $\Omega$.

DEFINITION 2.5. A spin integral cohomology class $x$ is an integral cohomology class for which $S q^{2} x=0$.

The fiber $E_{n}$ of the map

$$
K(Z, n) \stackrel{S q^{2} \iota_{n}}{\longrightarrow} K\left(Z_{2}, n+2\right)
$$

is a classifying space for spin integral classes of dimension $n$. We regard $E_{n}$ as $\Omega^{m} E_{n+m}$ and $e_{n}=\sigma^{m}\left(e_{n+m}\right)$ where $e_{j}$ is the fundamental class of $E_{j}$ and $\sigma$ is the suspension homomorphism.

Definition 2.6. A class $z$ in $H^{*}\left(E_{n}\right)$ is called stable if, for every positive integer $m$, there is a class $y$ in $H^{*}\left(E_{n+m}\right)$ such that $\sigma^{m}(y)=z$.

Set $E=E_{4 t+1}$ with fundamental class $e$. Note that $(0,0) \in \Omega(e)$ for $\Omega$ as chosen above. Consider the following stable relation on spin integral classes:

$$
S q^{2} \Omega_{1}+S q^{1} \Omega_{2}=0 .
$$

THEOREM 2.8. A stable tertiary operation $\psi$ associated to relation (2.7) can be chosen so that $\lambda e \cdot y \in \psi(e)$ where y generates $H^{4 t+4}(E)$ and $\lambda$ is in $Z_{2}$.

Proof. The universal example for a tertiary operation associated to (2.7) is a fiber space over $E$. Thus any choice for $\psi$ can be altered by stable classes in $H^{8 t+5}(E)$. 
It has a vector space basis over $Z_{2}$ consisting of stable classes $S q^{I} e$ for certain admissible monomials $S q^{I}$ in $A$ and also the nonstable class $e \cdot y$ where $y$ generates $H^{4 t+4}(E)$. This follows from the Serre spectral sequence applied to the fibration $r: E \rightarrow K(Z, 4 t+1)$ with fiber $K\left(Z_{2}, 4 t+2\right)$ and classifying map $S q^{2} \iota$. The result follows.

REMARK. It follows that $\lambda=1$ by a result of L. Kristensen. An immediate consequence of $(2.8)$ is the following.

Corollary 2.9. Let $X$ be a complex such that $H^{4 t+4}(X)=0$. A stable tertiary operation $\psi$ associated to relation (2.7) can be chosen, independently of $X$, so that $0 \in \psi(x)$ for every spin integral class $x$ in $H^{s}(X)$ for $s \leqq 4 t+1$.

In $\S 4$ it is necessary to evaluate a stable secondary operation on 1-dimensional classes. Recall that the excess of a homogeneous element $\theta$ in the Steenrod algebra $A$, written ex $(\theta)$, is the minimum value of the excesses of the admissible monomials in $A$ whose sum is $\theta$. Consider the following relation in $A$ of degree $n$ :

$$
S q^{1} \theta+\sum_{i=1}^{s} \gamma_{i} \theta_{i}=0
$$

where ex $\left(\theta_{i}\right)>1$ and degree $\left(\gamma_{i}\right)>1$ for $1 \leqq i \leqq s$. Let $\Omega$ be any stable secondary operation associated to $(2.10)$ and let $\rho$ denote $\bmod 2$ reduction of integral classes.

Proposition 2.11. Let $X$ be a complex and $x$ a class in $H^{1}(X)$ in the domain of $\Omega$ such that $x^{n}=0$. If $\operatorname{ex}(\theta)>1,0 \in \Omega(x)$. If $\operatorname{ex}(\theta)=1$, then $n=2^{r}$ for some integer $r$ and $\rho(u) \in \Omega(x)$ where $2 u=y^{2^{r-1}}$ in $H^{n}(X ; Z)$ and $\rho(y)=x^{2}$.

Proof. Let $f: X \rightarrow R P^{\infty}$ classify $x$ and let $\alpha$ denote the generator of $H^{*}\left(R P^{\infty}\right)$. If $\operatorname{ex}(\theta)>1$, the functional cohomology operation associated to (2.10) vanishes on $\alpha$ by [2, Teorema 6.6]. It follows from the Peterson-Stein formula [2, Teorema 5.2] and the assumption $x^{n}=0$ that $0 \in \Omega(x)$. If ex $(\theta)=1$, clearly $n=2^{r}$ for some integer $r$ and $\theta(\alpha)=\alpha^{2}$. Consider the following commutative diagram.

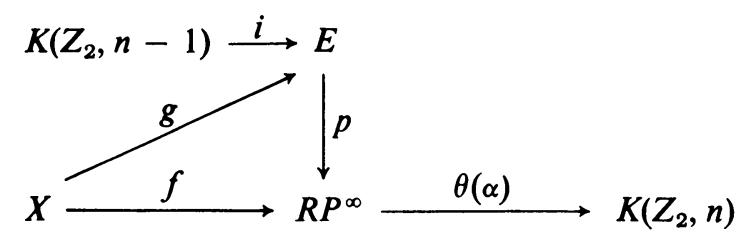

Here $p$ is the principal fiber map classified by the map $\theta(\alpha)$. Since $\alpha^{2}=\rho(\beta)$ for $\beta$ in $H^{2}\left(R P^{\infty} ; Z\right), p^{*} \beta^{2^{r-1}}=2 z$ for $z$ in $H^{n}(E ; Z)$. Further, $i^{*} \rho(z)=S q^{1} \iota$ since this is true in the universal example for division by 2. (See [9].) Applying the Serre spectral sequence to the fiber map $p$ shows that $H^{n}(E)$ is generated by $\rho(z)$. Set $y=f^{*} \beta$ and $2 u=y^{2^{r-1}}$. The universal example for $\Omega$ on 1-dimensional classes is a fiber space over $R P^{\infty}$ fiber homotopically equivalent to $E \times \prod_{i=1}^{s} K\left(Z_{2}\right.$, degree $\left.\theta_{i}\right)$. It follows that $\rho(u)=g^{*} \rho(z) \in \Omega(x)$. 
COROLlARY 2.13. Let $\Omega$ be a stable secondary operation associated to relation (2.10) with $n$ even. Let $M^{n}$ be an orientable manifold. Then any class $u$ in $H^{1}(M)$ lies in the domain of $\Omega$ and $0 \in \Omega(u)$.

Proof. Since $M$ is orientable, $u^{n}=S q^{1} u^{n-1}=0$. The result follows from (2.11) and the fact $H^{n}(M ; Z)=Z$.

3. Immersions of $k$-connected manifolds. In this section we derive some immersion results for certain $k$-connected manifolds for small values of $k$.

Proposition 3.1. Let $K$ be a complex of dimension $n \equiv 6 \bmod 8$ with $n>6$. Assume that $H^{n-1}(K)=H^{n-2}(K)=0$ and $S q^{1} H^{n-4}(K) \subseteq S q^{2} H^{n-5}(K)$. Let $\xi$ be a stable spin bundle over $K$ with $w_{n-6}(\xi)=0$. Then g.dim $\xi \leqq n-5$.

Proof. Set $n=8 t+6$ for $t>0$ and refer to Postnikov resolution III in $\S 5$. Now $w_{8 t+2}(\xi)=w_{8 t+4}(\xi)=0$ from the $\mathrm{Wu}$ relations since $w_{8 t}(\xi)=0$. Let the map $\xi: K \rightarrow B$ Spin classify the bundle $\xi$. Thus $k_{1}^{1}(\xi)$ is defined and $\xi$ clearly lifts to $B$ Spin $(8 t+1)$ iff $0 \in k_{1}^{1}(\xi)$. Note that $k_{1}^{1}$ is independent of $w_{8 t+4}$. One checks that $w_{8 t}$ in $H^{*}(B$ Spin $)$ is a generating class for $k_{1}^{1}$ with respect to the relation

$$
S q^{2} S q^{2}+S q^{1}\left(S q^{2} S q^{1}\right)=0
$$

Any secondary operation $\Omega$ associated to (3.2) is spin trivial since $B$ Spin is 3connected. By [26] for any choice of $\Omega, 0 \in \Omega\left(w_{8 t}\right) \subseteq H^{8 t+3}(B \operatorname{Spin}(8 t+1))$ since $\operatorname{ker} i^{*} \cap H^{8 t+3}(B \operatorname{Spin}(8 t+1))=0$ where $i: B \operatorname{Spin}(8 t) \subset B$ Spin $(8 t+1)$. Set $L$ $=\operatorname{Indet}^{8 t+3}(B$ Spin; $\Omega)$. Then $k_{1}^{1} \in \Omega\left(p_{1}^{*} w_{8 t}\right)$ by Theorem 2.3. The indeterminacy of $k_{1}^{1}(\xi)=\operatorname{Indet}^{8 t+3}(K ; \Omega)$ since by hypothesis $S q^{1} H^{8 t+2}(K) \subseteq S q^{2} H^{8 t+1}(K)$. Thus $0 \in \Omega\left(w_{8 t}(\xi)\right)=k_{1}^{1}(\xi)$.

THEOREM 3.3. Let $M^{n}$ be a 2-connected manifold with $n \equiv 6 \bmod 8$ and $n>6$. Assume $H_{3}(M ; Z)$ has no 2-torsion. Then $M \subseteq R^{2 n-5}$ and $M \subset R^{2 n-4}$.

Proof. Let $v$ denote the stable normal bundle of $M$. By $(1.2) w_{n-6}(v)=0 . H^{n-2}(M)$ $=H^{n-1}(M)=0$ and $H^{n-3}(M ; Z)$ has no 2-torsion by Poincaré duality so $S q^{1} H^{n-4}(M)=0$. Thus g.dim $v \leqq n-5$ by (3.1) and so $M \subseteq R^{2 n-5}$ by Hirsch [12]. $M \subset R^{2 n-4}$ by Glover [10].

THEOREM 3.4. Let $M^{n}$ be a 3-connected manifold with $n \equiv 7 \bmod 8$ and $n>7$. Suppose $S q^{1} H^{n-5}(M) \subseteq S q^{2} H^{n-6}(M)$. Then $M \subseteq R^{2 n-6}$.

Proof. Write $n=8 t+7$ for $t>0$ and refer to resolution III in $\S 5$. Let $v: M \rightarrow B$ Spin classify the stable normal bundle of $M$. By $(1.2) w_{8 t}(v)=0$ so $w_{8 t+2}(v)=0$ from the $\mathrm{Wu}$ relations. Clearly $v$ lifts to $B$ Spin $(8 t+1)$ iff $0 \in k_{1}^{1}(v)$ and $k_{4}^{1}(v)=0$. The proof of (3.1) shows that $0 \in k_{1}^{1}(v)$.

Note that $k_{4}^{1}$ is independent of $w_{8 t+2}$. Let $U_{v}$ and $T_{v}$ denote the Thom class and 
Thom complex of $v$ respectively. By [15] we can choose a stable secondary operation $\Gamma$ associated to the relation

$$
S q^{4} S q^{8 t+4}+S q^{8 t+7} S q^{1}+S q^{8 t+6} S q^{2}=0
$$

such that $u \cdot S q^{4} u \in \Gamma(u)$ for any class $u$ of dimension $8 t+3$ in the domain of $\Gamma$. Applying the technique for isolating an independent $k$-invariant from a resolution in [28] and the generating class theorem [28, Theorem 6.5] gives the result $U_{v} \cdot k_{4}^{1}(v)=\Gamma\left(U_{v}\right)$ in $H^{*}\left(T_{v}\right)$. But the top class in $H^{*}\left(T_{v}\right)$ is spherical by [16] so $\Gamma\left(U_{v}\right)=0$. Thus g.dim $v \leqq 8 t+1$ and the result follows by [12].

Let $M^{n}=S^{3} \times C P^{2^{r+1}}$ for $r>1$. It follows from [7] that $M \nsubseteq R^{2 n-7}$ so the following result is best possible.

THEOREM 3.5. Let $M^{n}$ be a simply connected spin manifold with $n \equiv 5 \bmod 8$ and $n>13$. Suppose the following conditions hold:

1. $x^{2}=0$ iff $x=0$ for any $x$ in $H^{2}(M)$.

2. $y^{2}=0$ iff $S q^{2} y=0$ for any $y$ in $H^{3}(M)$.

3. $\bar{w}_{n-6}(M)=0$ if $n=2^{r}+5$.

Then $M$ immerses in $R^{2 n-6}$.

Proof. Write $n=8 t+5$ for $t>1$ and refer to Postnikov resolution IV in $\S$. Let $v: M \rightarrow B$ Spin classify the stable normal bundle of $M$. Note that $w_{8 t}(v)=0$ by $(1.2)$ so $v$ lifts to $E_{1}$. A simple argument using Poincaré duality and the Wu classes shows that $S q^{2}: H^{n-4}(M) \rightarrow H^{n-2}(M)$ is an epimorphism iff condition 1 holds. If $v$ lifts to $E_{2}, 0 \in k_{1}^{2}(v)$ since the indeterminacy subgroup of $k_{1}^{2}(v)=S q^{2} H^{n-4}(M)$. Since $M$ is simply connected, $v$ lifts to $B \operatorname{Spin}(8 t-1)$ iff $0 \in k_{1}^{1}(v)$ and $k_{3}^{1}(v)=0$.

The functional cohomology operation associated to the relation

$$
\left(S q^{4} S q^{2}\right) S q^{8 t}+S q^{8 t+4} S q^{2}+S q^{8 t+3}\left(S q^{2} S q^{1}\right)=0
$$

vanishes on classes of dimension $<8 t$ in its domain by [2, Teorema 6.6]. By the Peterson-Stein formula [2, Teorema 5.2] a stable secondary operation $\Gamma$ associated to (3.6) can be chosen independently of $u$ so that $\lambda u \cdot S q^{6} u \in \Gamma(u)$ for fixed $\lambda$ in $Z_{2}$ where $u$ is any class of dimension $8 t-1$ in the domain of $\Gamma$. Applying the generating class theorem [28, Theorem 6.5] gives

$$
U_{E_{1}} \cdot\left(k_{3}^{1}+\lambda p_{1}^{*}\left(w_{6} w_{8 t-1}\right)\right) \in \Gamma\left(U_{E_{1}}\right) .
$$

Note that $S q^{2}\left(w_{4} \cdot w_{8 t-1}\right)=w_{6} w_{8 t-1}+w_{4} w_{8 t+1}$ so $w_{6}(v) w_{8 t-1}(v)=0$. Thus $U_{v} \cdot k_{3}^{1}(v)$ $=\Gamma\left(U_{v}\right)$ since $\Gamma\left(U_{v}\right)$ has zero indeterminacy. But the top class in $H^{*}\left(T_{v}\right)$ is spherical by [16] so $k_{3}^{1}(v)=0$.

One checks that $w_{8 t-2}$ in $H^{*}(B$ Spin $)$ is a generating class for $k_{1}^{1}$ with respect to the relation

$$
S q^{2}\left(S q^{2} S q^{1}\right)=0
$$

Let $\Omega$ be the spin trivial stable secondary operation associated to (3.7). (See [26].) By [26, Theorem 3.3] (or Remark 2 in $\S 2) 0 \in \Omega\left(w_{8 t-2}\right)$ in $H^{*}(B$ Spin $(8 t-1)$ ) since 
$\operatorname{ker} i^{*} \cap H^{8 t+2}(B \operatorname{Spin}(8 t-1))=0$ where $i: B$ Spin $(8 t-2) \subset B$ Spin $(8 t-1)$. Thus $k_{1}^{1} \in \Omega\left(p_{1}^{*} w_{8 t-2}\right)$ by the generating class theorem [25, Theorem 5.9]. It follows from Poincaré duality and the Wu classes that condition 2 holds iff $S q^{2} H^{8 t}(M)$ $=S q^{2} S q^{1} H^{8 t-1}(M)$. So $k_{1}^{1}(v)=\Omega\left(w_{8 t-2}(v)\right)$. But from [26] $\Omega=\varphi \circ \delta$ where $\varphi$ is the unique secondary operation associated to the integral relation $S q^{2} S q^{2}=0$ and $\delta$ is the Bockstein operator. Since $\bar{w}_{n-6}(M)=0$ from [18] and condition 3, it follows that $0 \in \varphi\left(\bar{w}_{n-6}(M)\right)=\varphi\left(\delta w_{8 t-2}(v)\right)=\Omega\left(w_{8 t-2}(v)\right)=k_{1}^{1}(v)$. So g.dim $v \leqq n-6$ and the result follows by Hirsch [12].

4. Orientable and spin manifolds. In this section we establish immersions for some orientable and spin manifolds. $Q P^{n}$ has a best possible immersion in $R^{8 n-3}$ for $n=2^{r}$ by [16]. $C P^{m}$ does not immerse in $R^{4 m-3}$ for $m=2^{r}+2^{s}$ with $r>s>0$ by [22]. For spin manifolds we prove the following

THEOREM 4.1. Let $M^{n}$ be a spin manifold with $n \equiv 0 \bmod 4$. Then $M$ immerses in $R^{2 n-3}$ for $n$ not a power of 2. $M$ immerses in $R^{2 n-3}$ iff $\bar{w}_{n-2}(M)=0$ for $n=2^{r}$ with $r>3$.

Proof. Set $n=4 t+4$ for $t>1$ and refer to Postnikov resolution $\mathrm{I}$ in $\S 5$. Let $v: M \rightarrow B$ Spin classify the stable normal bundle $v$ of $M$. Now $w_{4 t+2}(v)=w_{4 t+4}(v)$ $=0$ by $(1.1)$ and the assumption $\bar{w}_{n-2}(M)=0$ for $n=2^{r}$. Note that $k_{1}^{1}(v)$ and $k_{2}^{1}(v)$ have zero indeterminacy. Let $U_{4 t+1}$ denote the Thom class associated to the universal bundle $\gamma_{4 t+1}$ over $B$ Spin $(4 t+1)$. Let $\Omega=\left(\Omega_{1}, \Omega_{2}\right)$ be the double secondary operation associated to relation $(2.4)$ such that $(0,0) \in \Omega(e)$. (See $\S 2$.) Thus $(0,0) \in \Omega\left(U_{4 t+1}\right)$. Let $T_{v}$ and $U_{v}$ denote the Thom complex and Thom class associated to $v$ respectively. Applying a version of the generating class theorem [27, Theorem 6.4] for expressing simultaneously two second-order $k$-invariants lifted to the Thom complex and then checking indeterminacies gives the result that $U_{v} \cdot\left(k_{1}^{1}(v), k_{2}^{1}(v)\right)=\Omega\left(U_{v}\right)$. (See also [21].) But $U_{v} \cdot k_{2}^{1}(v)=\Omega_{2}\left(U_{v}\right)=0$ since the top class in $H^{*}\left(T_{v}\right)$ is spherical by [16]. We apply a duality theorem of Adem-Gitler [3, Theorem 5.1] in order to show $k_{1}^{1}(v)=0$. Let $\Gamma$ denote the secondary operation dual to $\Omega_{1}$ and associated to the relation

$$
c\left(S q^{4 t+2}\right) S q^{2}+S q^{1} c\left(S q^{4 t+3}\right)=0
$$

where $c$ is the anti-automorphism of $A$. Then $\Omega_{1}\left(U_{v}\right)=0$ iff $\Gamma$ vanishes on its domain of definition in $H^{1}(M)$ from [3]. By (2.13) $\Gamma$ vanishes on every class in $H^{1}(M)$ so $k_{1}^{1}(v)=0$.

The $k$-invariant $k_{1}^{2}$ can be expressed by the tertiary operation $\psi$ of Theorem 2.8 . Since $B$ Spin $(4 t+1)$ is 3-connected, $0 \in \psi\left(U_{4 t+1}\right)$ by $(2.9)$. Note that $k_{1}^{2}(v)$ has zero indeterminacy. Applying a version of the generating class theorem for a third-order $k$-invariant lifted to the Thom complex [21, Proposition 4.6] gives the result $U_{v} \cdot k_{1}^{2}(v) \in \psi\left(U_{v}\right)$. But $\psi\left(U_{v}\right)=0$ since the top class in $H^{*}\left(T_{v}\right)$ is spherical by [16] so $k_{1}^{2}(v)=0$. Thus $v$ lifts to $B$ Spin $(4 t+1)$ and the result follows by Hirsch [12]. 
REMARK. It follows from [31, Lemma 1] that a 4-dimensional spin manifold immerses in $R^{5}$.

Thomas proves in [26] that a spin manifold $M^{n}$ immerses in $R^{2 n-4}$ for $n \equiv 3 \bmod 8$ and $n>3$.

THEOREM 4.3. Let $M^{n}$ be a spin manifold with $n \equiv 7 \bmod 8$ and $n>7$. Let $\xi$ be a stable spin bundle over $M$. If $w_{n-7}(\xi)=0$, g.dim $\xi \leqq n-4$. Thus $M \subseteq R^{2 n-4}$.

Proof. Set $n=8 t+7$ and refer to resolution II in $\S 5$. Let $\xi: M \rightarrow B$ Spin classify the bundle $\xi$. Now $w_{8 t+4}(\xi)=0$ since $w_{8 t+4}=S q^{4} w_{8 t}+w_{4} \cdot w_{8 t}$ in $H^{*}(B$ Spin $)$. We express $k_{2}^{1}$ by means of a twisted secondary operation due to Thomas. Consider the following relation in $A\left(K\left(Z_{2}, 4\right)\right)$ :

$$
\gamma \cdot \gamma+S q^{2}\left(\gamma \cdot S q^{2}\right)+S q^{1}\left(S q^{2} \gamma S q^{1}\right)+\delta \cdot\left(S q^{2} S q^{1}\right)=0
$$

where $\gamma=\imath \otimes 1+1 \otimes S q^{4}$ and $\delta=S q^{1} \iota \otimes 1$. Let $w_{4}: B$ Spin $\rightarrow K\left(Z_{2}, 4\right)$ classify the Stiefel-Whitney class $w_{4}$. One checks that $w_{8 t}$ in $H^{*}(B$ Spin $)$ is a generating class for $k_{2}^{1}$ with respect to the relation (4.4). Let $\varphi$ be a secondary operation associated to (4.4). Let $U_{s}$ denote the Thom class associated to the universal bundle $\gamma_{s}$ over $B$ Spin $(s)$ for $s>7$. Clearly $\varphi\left(U_{s}, w_{4}\right)$ is defined and $\varphi$ is spin trivial since $U_{s} \cdot w_{7}$ $=S q^{1}\left(U_{s} \cdot w_{6}\right)$. Let $j: B$ Spin $(8 t) \subset B$ Spin $(8 t+3)$ denote the standard inclusion. Since $\operatorname{ker} j^{*} \cap H^{8 t+7}(B \operatorname{Spin}(8 t+3))$ is generated by $w_{6} w_{8 t+1}$ and $w_{4} w_{8 t+3}$, it follows from Remark 2 in $\$ 2$ that

$$
\lambda_{1} w_{6} w_{8 t+1}+\lambda_{2} w_{4} w_{8 t+3} \in \varphi\left(w_{8 t}, w_{4}\right)
$$

in $H^{8 t+7}(B$ Spin $(8 t+3))$ for some $\lambda_{1}$ and $\lambda_{2}$ in $Z_{2}$. Since $S q^{5} S q^{2} w_{8 t}=w_{4} w_{8 t+3}$ and $S q^{2}\left(w_{4} \cdot S q^{1} w_{8 t}\right)=w_{6} w_{8 t+1}$, one has $0 \in \Gamma\left(w_{8 t}, w_{4}\right)$ in $H^{*}(B \operatorname{Spin}(8 t+3))$ for

$$
\Gamma=\varphi+\lambda_{1}\left(1 \otimes S q^{5} S q^{2}\right)+\lambda_{2} S q^{2}\left(\iota \otimes S q^{1}\right) .
$$

The generating class theorem [25, Theorem 5.9] gives $k_{2}^{1} \in \Gamma\left(p_{1}^{*} w_{8 t}, p_{1}^{*} w_{4}\right)$. Now $\operatorname{Indet}^{8 t+7}\left(M ; \Gamma, w_{4}(\xi)\right)=\left(S q^{4}+\cdot w_{4}(\xi)\right) H^{n-4}(M)=$ indeterminacy of $k_{2}^{1}(\xi)$. Thus $0 \in \Gamma\left(w_{8 t}(\xi), w_{4}(\xi)\right)=k_{2}^{1}(\xi)$. The proof of Theorem 1.3 in [26] shows that $k_{1}^{1}(\xi)$ $=k_{1}^{2}(\xi)=0$. Thus $\xi$ lifts to $B$ Spin $(8 t+3)$. By $(1.2) \bar{w}_{n-7}(M)=0$ so g.dim $v \leqq n-4$ where $v$ denotes the stable normal bundle of $M$.

Theorem 4.3 has an immediate application to a problem investigated by Thomas in [29]. Here we require a manifold $M$ to mean only a smooth connected manifold without boundary. Let $\tau_{0}(M)$ and $v(M)$ denote the stable tangent and normal bundles of a manifold $M$ respectively. Given a map $f: M \rightarrow N$ between manifolds, define the stable bundle $v_{f}=f^{*} \tau_{0}(N)+v(M)$. The map $f$ is called a spin map if $f^{*} w_{1}(N)=f^{*} w_{2}(N)=0$ and $M$ is a closed spin manifold.

THEOREM 4.5. Let $M^{8 t+7}$ and $N^{16 t+10}$ be manifolds with $t>0$. Suppose $f: M \rightarrow N$ is a spin map. If $w_{8 t}\left(v_{f}\right)=0$, then $f$ is homotopic to an immersion. 
Proof. Note that $v_{f}$ is a stable spin bundle over $M$. By (4.3) g.dim $v_{f} \leqq 8 t+3$ $=\operatorname{dim} N-\operatorname{dim} M$. The result follows from the formulation of Hirsch's theorem in [29].

Manifolds again are assumed to be closed. We prove

THEOREM 4.6. Let $M^{n}$ be an orientable manifold with $n \equiv 1 \bmod 4$ and $n>9$. Suppose the following conditions hold:

1. $u^{2}=0$ iff $u=0$ for any $u$ in $H^{1}(M)$.

2. $w_{2}(M)=u^{2}$ for some $u$ in $H^{1}(M)$ iff $w_{2}(M)=0$.

3. $S q^{1} y=0$ for any $y$ in $H^{2}(M)$ such that $y^{2}=0$.

4. $\bar{w}_{n-5}(M)=0$ if $\alpha(n)<5$.

Then $M$ immerses in $R^{2 n-4}$.

Proof. Write $n=4 t+5$ and refer to Postnikov resolution V in $\S 5$. Let $v: M \rightarrow B$ classify the stable normal bundle $v$ of $M$.

Case I. $B=B S O$ and $w_{2}(M) \neq 0$.

Condition 4 and [18] give $w_{4 t+2}(v)=w_{4 t+4}(v)=0$. Condition 1 is equivalent to $S q^{1} H^{4 t+3}(M)=H^{4 t+4}(M)$ from Poincaré duality and the Wu relations. So $0 \in k_{2}^{1}(v)$ since $S q^{1} H^{4 t+3}(M)=$ indeterminacy of $k_{2}^{1}(v)$. Note that $0 \in k_{1}^{2}(v)$ also through indeterminacy if $v$ lifts to $E_{2}$. Let $g: M \rightarrow E_{1}$ be a lifting of $v$ such that $g^{*} k_{2}^{1}=0$. Condition 2 is equivalent to the condition $S q^{2} y \neq 0$ and $S q^{1} y=0$ for some class $y$ in $H^{n-2}(M)$. Alter $g$, if necessary, to give a lifting $h: M \rightarrow E_{1}$ of $v$ such that $h^{*} k_{3}^{1}=0$ and $h^{*} k_{2}^{1}=g^{*} k_{2}^{1}=0$. Note that

$$
\left(S q^{2}+\cdot w_{2}(M)\right) S q^{1} H^{n-4}(M)=0=\left(S q^{4}+\cdot \bar{w}_{4}(M)\right) H^{n-4}(M) .
$$

Thus $v$ lifts to $B S O(4 t+1)$ iff $0 \in k_{1}^{1}(v)$. Assume that $\alpha(n)>4$. Let $\varphi$ be the secondary operation associated to the relation- $S q^{2} S q^{4 t+2}+S q^{4 t+3} S q^{1}=0$-such that $u \cdot S q^{2} u \in \varphi(u)$ for any class $u$ of dimension $4 t+1$ in the domain of $\varphi$. The generating class theorem [28, Theorem 6.5] gives the result $U_{E_{1}} \cdot\left(k_{1}^{1}+p_{1}^{*} w_{2} w_{4 t+1}\right) \in \varphi\left(U_{E_{1}}\right)$. But $w_{4 t+1}(v)=0$ for $\alpha(n)>4$ by [18] so $U_{v} \cdot k_{1}^{1}(v)=\varphi\left(U_{v}\right)$. Let $\Gamma$ denote the operation dual to $\varphi$ associated to the relation (4.2). By [3] $\varphi$ vanishes on $U_{v}$ iff $\Gamma$ vanishes on its domain of definition in $H^{2}(M)$. Recall from [18] that a homogeneous element $\theta$ of degree $r-s$ in the Steenrod algebra $A$ vanishes on $s$-dimensional classes if $\alpha(r)>s$. Thus the functional cohomology operation $\psi$ associated to relation (4.2) vanishes on 2-dimensional classes since $\alpha(4 t+5)>4$. (See [2].) It follows from condition 3 and the Peterson-Stein formula in [2] that $\Gamma(u)=\psi(u)$ for any class $u$ in $H^{2}(M)$ in the domain of $\Gamma$. So $0 \in k_{1}^{1}(v)$ for $\alpha(n)>4$.

Suppose now that $\alpha(n)<5$. Consider the relation in $A\left(K\left(Z_{2}, 2\right)\right)$ :

$$
\beta \cdot \beta+S q^{1} \cdot\left(\beta \cdot S q^{1}\right)=0
$$

where $\beta=\imath \otimes 1+1 \otimes S q^{2}$. Let the map $w_{2}: B S O \rightarrow K\left(Z_{2}, 2\right)$ induce an $A\left(K\left(Z_{2}, 2\right)\right)$ module structure on $H^{*}(B S O)$. By $\S 6$ of [25] a twisted secondary operation $\Omega$ 
associated to (4.7) can be chosen so that $k_{1}^{1} \in \Omega\left(p_{1}^{*} w_{4 t}, p_{1}^{*} w_{2}\right)$. Condition 3 is equivalent to the condition $S q^{1} H^{n-3}(M) \subseteq\left(S q^{2}+\cdot w_{2}(M)\right) H^{n-4}(M)$. So

$$
0 \in \Omega\left(w_{4 t}(v), w_{2}(v)\right)=k_{1}^{1}(v)
$$

by condition 4 . Thus $v$ lifts to $B S O(4 t+1)$.

Case II. $B=B$ Spin and $w_{2}(M)=0$. The only essential difference from Case $I$ is the computation of $k_{3}^{1}(v)$. We choose by [15] the secondary operation $\Gamma$ associated to the stable integral relation

$$
S q^{4} S q^{4 t+2}+S q^{4 t+4} S q^{2}+t S q^{2} S q^{4 t+4}=0
$$

such that $u \cdot S q^{4} u \in \Gamma(u)$ for any spin integral class $u$ of dimension $4 t+1$. By the generating class theorem $U_{E_{1}} \cdot\left(k_{3}^{1}+p_{1}^{*} w_{4} w_{4 t+1}\right) \in \Gamma\left(U_{E_{1}}\right) . S q^{1}\left(w_{4} w_{4 t}\right)=w_{4} w_{4 t+1}$ so $w_{4}(v) \cdot w_{4 t+1}(v)=0$. Thus $0=\Gamma\left(U_{v}\right)=U_{v} \cdot k_{3}^{1}(v)$ since the top class in $H^{*}\left(T_{v}\right)$ is spherical by [16]. So $k_{3}^{1}(v)=0$ and the result follows.

Refer to [6] and [32] for the cohomology ring and total Stiefel-Whitney class of the Dold manifold $P(m, n)$. A consequence of Theorem 4.6 is the following

Corollary 4.9. Set $N=m+2 n$. Let $P(m, n)$ be any orientable Dold manifold with $N \equiv 1 \bmod 4, m>1, n>0$, and $n \neq 2^{r}$ when $\alpha(N) \leqq 3$. Then $P(m, n) \subseteq R^{2 N-4}$.

5. Postnikov resolutions. These Postnikov resolutions for the fiber map $\pi: B_{m} \rightarrow B$ are constructed by the techniques of [24]. We refer the reader also to [14] and [8] for the theory and construction of modified Postnikov resolutions. The homotopy groups of the fiber for $\pi$ appear in [13] and [20]. The tower of spaces is displayed only for resolution I. The $k$-invariant $k_{j}^{i}$ represents a class in $H^{*}\left(E_{i}\right)$ whose defining relation is a relation in $H^{*}\left(E_{i-1}\right)$ where $E_{0}=B$.

5.1. Postnikov resolution I for the fibration $\pi: B$ Spin $(4 t+1) \rightarrow B$ Spin for stable spin bundles over complexes of dimension $\leqq 4 t+4$ for $t>1$.

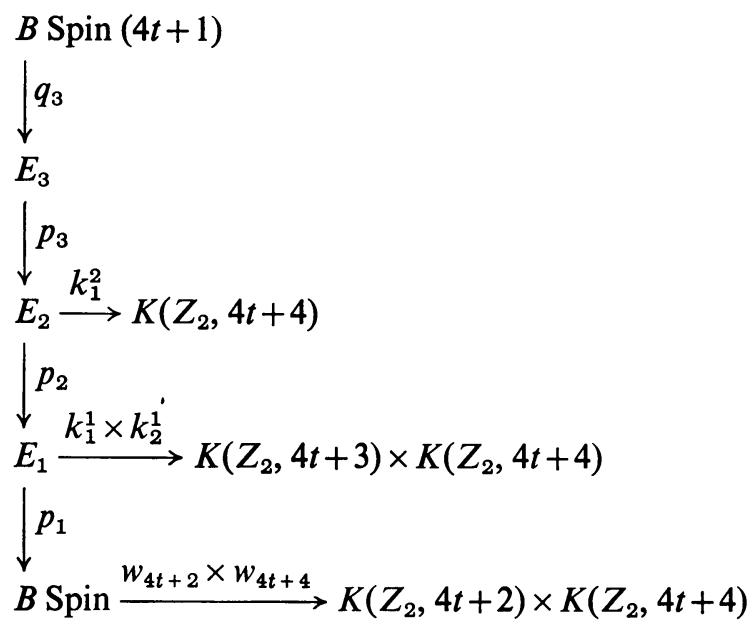


Defining relations for $k$-invariants:

$$
\begin{aligned}
& k_{1}^{1}: S q^{2} w_{4 t+2}=0, \\
& k_{2}^{1}: S q^{2} S q^{1} w_{4 t+2}+S q^{1} w_{4 t+4}=0, \\
& k_{1}^{2}: S q^{2} k_{1}^{1}+S q^{1} k_{2}^{1}=0 .
\end{aligned}
$$

5.2. Postnikov resolution II for the fibration $\pi: B$ Spin $(8 t+3) \rightarrow B$ Spin for stable spin bundles over complexes of dimension $\leqq 8 t+7$ for $t>0$.

Defining relations for $k$-invariants:

$$
\begin{aligned}
& k_{1}^{0}=w_{8 t+4}, \\
& k_{1}^{1}: S q^{2} S q^{1} w_{8 t+4}=0, \\
& k_{2}^{1}:\left(S q^{4}+\cdot w_{4}\right) w_{8 t+4}=0, \\
& k_{1}^{2}: S q^{2} k_{1}^{1}=0 .
\end{aligned}
$$

5.3. Postnikov resolution III for the fibration $\pi: B$ Spin $(8 t+1) \rightarrow B$ Spin for stable spin bundles over complexes of dimension $\leqq 8 t+7$ for $t>0$.

Defining relations for $k$-invariants:

$$
\begin{aligned}
& k_{1}^{0}=w_{8 t+2}, \quad k_{2}^{0}=w_{8 t+4}, \\
& k_{1}^{1}: S q^{2} w_{8 t+2}=0, \\
& k_{2}^{1}: S q^{2} S q^{1} w_{8 t+2}+S q^{1} w_{8 t+4}=0, \\
& k_{3}^{1}:\left(S q^{4}+\cdot w_{4}\right) w_{8 t+2}=0, \\
& k_{4}^{1}:\left(S q^{4}+\cdot w_{4}\right) w_{8 t+4}=0, \\
& k_{1}^{2}: S q^{2} k_{1}^{1}+S q^{1} k_{2}^{1}=0 .
\end{aligned}
$$

5.4. Postnikov resolution IV for the fibration $\pi: B$ Spin $(8 t-1) \rightarrow B$ Spin for stable spin bundles over complexes of dimension $\leqq 8 t+5$ for $t>1$. Defining relations for $k$-invariants:

$$
\begin{aligned}
& k_{1}^{0}=w_{8 t}, \\
& k_{1}^{1}: S q^{2} S q^{1} w_{8 t}=0, \\
& k_{2}^{1}:\left(S q^{4}+\cdot w_{4}\right) S q^{1} w_{8 t}=0, \\
& k_{3}^{1}:\left(S q^{4}+\cdot w_{4}\right) S q^{2} w_{8 t}=0, \\
& k_{1}^{2}: S q^{2} k_{1}^{1}=0, \\
& k_{2}^{2}: S q^{2} S q^{1} k_{1}^{1}+S q^{1} k_{2}^{1}=0, \\
& k_{1}^{3}: S q^{2} k_{1}^{2}+S q^{1} k_{2}^{2}=0 .
\end{aligned}
$$

5.5. Postnikov resolution $\mathrm{V}$ for the fibration $\pi: B(4 t+1) \rightarrow B$ for stable orient- 
able and spin bundles over complexes of dimension $\leqq 4 t+5$ for $t>1$. Defining relations for $k$-invariants:

$$
\begin{aligned}
& B=B S O, \\
& k_{1}^{0}=w_{4 t+2}, \\
& k_{2}^{0}=w_{4 t+4}, \\
& k_{1}^{1}:\left(S q^{2}+\cdot w_{2}\right) w_{4 t+2}=0, \\
& k_{2}^{1}:\left(S q^{2}+\cdot w_{2}\right) S q^{1} w_{4 t+2}+S q^{1} w_{4 t+4}=0, \\
& k_{3}^{1}:\left(S q^{4}+\cdot w_{4}\right) w_{4 t+2}+S q^{2} w_{4 t+4}=0, \quad t \text { odd, } \\
& k_{3}^{1}:\left(S q^{4}+\cdot w_{4}\right) w_{4 t+2}+w_{2} w_{4 t+4}=0, \quad t \text { even, } \\
& k_{1}^{2}:\left(S q^{2}+\cdot w_{2}\right) k_{1}^{1}+S q^{1} k_{2}^{1}=0 .
\end{aligned}
$$

The $k$-invariants for $B=B$ Spin are obtained by deleting $w_{2}$ in the above defining relations.

\section{REFERENCES}

1. J. F. Adams, On the non-existence of elements of Hopf invariant one, Ann. of Math. (2) 72 (1960), 20-104. MR 25 \#4530.

2. J. Adem, On secondary cohomology operations, Bol. Soc. Mat. Mexicana (2) 7 (1962), 95-110. (Spanish) MR 29 \#5243.

3. J. Adem and S. Gitler, Secondary characteristic classes and the immersion problem, Bol. Soc. Mat. Mexicana (2) 8 (1963), 53-78. MR 29 \#5255.

4. J. Adem, S. Gitler and M. Mahowald, Embedding and immersion of projective spaces, Bol. Soc. Mat. Mexicana (2) 10 (1965), 84-88. MR 36 \#3369.

5. M. F. Atiyah, Thom complexes, Proc. London Math. Soc. (3) 11 (1961), 291-310. MR 24 \#A1727.

6. A. Dold, Erzeugende der Thomschen Algebra $\mathfrak{N}$, Math. Z. 65 (1956), 25-35. MR 18, 60.

7. S. Feder, Non-immersion theorems for complex and quaternionic projective spaces, Bol. Soc. Mat. Mexicana (2) 11 (1966), 62-67. MR 38 \#721.

8. S. Gitler and M. Mahowald, The geometric dimension of real stable vector bundles, Bol. Soc. Mat. Mexicana (2) 11 (1966), 85-107. MR 37 \#6922.

9. S. Gitler, M. Mahowald and R. J. Milgram, Secondary cohomology operations and divisibility of the Chern class, Proc. Amer. Math. Soc. (to appear).

10. H. Glover, Thesis, University of Michigan, Ann. Arbor, Mich., 1967.

11. A. Haefliger and M. W. Hirsch, On the existence and classification of differentiable embeddings, Topology 2 (1963), 129-135. MR 26 \#6981.

12. M. W. Hirsch, Immersion of manifolds, Trans. Amer. Math. Soc. 93 (1959), 242-276. MR 22 \#9980.

13. C. S. Hoo and M. E. Mahowald, Some homotopy groups of Stiefel manifolds, Bull. Amer. Math. Soc. 71 (1965), 661-667. MR 31 \#1675.

14. M. E. Mahowald, On obstruction theory in orientable fiber bundles, Trans. Amer. Math. Soc. 110 (1964), 315-349. MR 28 \#620.

15. - Some Whitehead products in $S^{n}$, Topology 4 (1965), 17-26. MR 31 \#2724.

16. M. E. Mahowald and F. P. Peterson, Secondary cohomology operations on the Thom class, Topology 2 (1963), 367-377. MR 28 \#612.

17. M. E. Mahowald and R. F. Williams, The stable homotopy of $K(Z, n)$, Bol. Soc. Mat. Mexicana (2) 11 (1966), 22-28. MR 38 \#3867. 
18. W. S. Massey and F. P. Peterson, On the dual Stiefel-Whitney classes of a manifold, Bol. Soc. Mat. Mexicana (2) 8 (1963), 1-13. MR 29 \#628.

19. - The cohomology structure of certain fibre spaces. I, Topology 4 (1965), 47-65. MR 32 \#6459.

20. G. F. Paechter, The groups $\pi_{r}\left(V_{n, m}\right)$. I, Quart. J. Math. Oxford Ser. (2) 7 (1956), 249-268. MR 24 \#A1725.

21. A. D. Randall, Some immersion theorems for projective spaces, Trans. Amer. Math. Soc. 147 (1970), 135-151.

22. B. J. Sanderson and R. L. E. Schwarzenberger, Non-immersion theorems for differentiable manifolds, Proc. Cambridge Philos. Soc. 59 (1963), 319-322. MR 26 \#5589.

23. E. H. Spanier, Algebraic topology, McGraw-Hill, New York, 1966. MR 35 \#1007.

24. E. Thomas, Seminar on fiber spaces, Lecture Notes in Math., no. 13, Springer-Verlag, Berlin and New York, 1966. MR 34 \#3582.

25. - Postnikov invariants and higher order cohomology operations, Ann. of Math. (2) 85 (1967), 184-217. MR 35 \#1029.

26. - Real and complex vector fields on manifolds, J. Math. Mech. 16 (1967), 1183-1205. MR 35 \#1030.

27. - The index of a tangent 2-field, Comment. Math. Helv. 42 (1967), 86-110. MR 35 \#6158.

28. - The span of a manifold, Quart. J. Math. Oxford Ser. (2) 19 (1968), 225-244. MR 38 \#2804.

29. —, On the existence of immersions and submersions, Trans. Amer. Math. Soc. 132 (1968), 387-394. MR 37 \#926.

30. ——, An exact sequence for principal fibrations, Bol. Soc. Mat. Mexicana (2) 12 (1967), 35-45. MR 39 \#4847.

31. — Vector fields on low dimensional manifolds, Math. Z. 103 (1968), 85-93. MR 36 \#7156.

32. J. J. Ucci, Immersions and embeddings of Dold manifolds, Topology 4 (1965), 283-293. MR 32 \#4703.

UNIVERSity OF NOTRE DAME,

Notre Dame, INDIANA 46556 